University of Nebraska - Lincoln

DigitalCommons@University of Nebraska - Lincoln

John R. Hardy Papers

Research Papers in Physics and Astronomy

$11-15-1984$

\title{
Model Calculations of the Micrometer to Millimeter Intrinsic Absorption for Simple Ceramics
}

John R. Hardy

University of Nebraska - Lincoln

P. J. Edwardson

University of Nebraska - Lincoln

J. A. O'Gara

University of Nebraska - Lincoln

Follow this and additional works at: https://digitalcommons.unl.edu/physicshardy

Part of the Physics Commons

Hardy, John R.; Edwardson, P. J.; and O'Gara, J. A., "Model Calculations of the Micrometer to Millimeter Intrinsic Absorption for Simple Ceramics" (1984). John R. Hardy Papers. 39.

https://digitalcommons.unl.edu/physicshardy/39

This Article is brought to you for free and open access by the Research Papers in Physics and Astronomy at DigitalCommons@University of Nebraska - Lincoln. It has been accepted for inclusion in John R. Hardy Papers by an authorized administrator of DigitalCommons@University of Nebraska - Lincoln. 


\title{
Model calculations of the micrometer to millimeter intrinsic absorption for simple ceramics
}

\author{
J. R. Hardy, P. J. Edwardson, and J. A. O'Gara \\ Behlen Laboratory of Physics, University of Nebraska, Lincoln, Nebraska 68588-0111
}

(Received 26 April 1984; revised manuscript received 18 June 1984).

\begin{abstract}
A systematic study has been made of the strength of the intrinsic absorption, or loss, for millimeter to micrometer electromagnetic radiation propagating in simple ceramic materials. This study employed a number of model diatomic systems whose simplicity enabled the basic two-phonon decay process, responsible for this intrinsic loss, to be computed with precision, even at the lowest frequencies, on a dedicated microcomputer. It was found that, at least at low frequencies, the loss was a relatively structureless universal function of the atomic mass ratio, in general agreement with earlier results for real alkali halides. In particular, it was found that, if one atomic mass was markedly increased, the low-frequency damping dropped dramatically and a "window" appeared in the loss function. This behavior and its origins are discussed and illustrated by predictions of the absolute values of the intrinsic low-frequency losses for $\mathrm{MgO}$ and $\mathrm{SrO}$ at room temperature. The values for $\mathrm{MgO}$ are almost an order of magnitude larger, and in reasonable accord with values measured for typical ceramics, when estimated extrinsic losses are subtracted. The present studies also provide a natural explanation of the general observation that the loss at the fundamental lattice resonance frequency is, at least in centrosymmetric systems, dominated by three-phonon decay.
\end{abstract}

\section{INTRODUCTION}

There has recently been a resurgence of interest in the optical properties of simple insulators at very long wavelengths; specifically, recent interest has centered on the millimeter to submillimeter region. This stems from recent developments in technology which have produced increasingly high-power sources operating in this region (e.g., gyrotons and free-electron lasers), and the consequent need to understand fully the interaction between such electromagnetic radiation and materials. This is important not only because of its intrinsic scientific interest, but also for very practical reasons, the most obvious of which is the need for reliable window materials in this spectral region. In the past, since practically any reasonably pure insulator is highly transparent at these frequencies, residual absorption has been unimportant and the choice among such materials has been dictated primarily by other criteria, such as mechanical strength. With the advent of these new sources of millimeter radiation, this is no longer the case. As the power level rises, so does the energy absorbed in the window: At some point this will become the limiting factor. This will occur when the rate of heat generation by absorption exceeds its rate of dissipation by thermal conduction and other mechanisms. Failure would appear to be an inherently catastrophic process since, beyond some critical heating rate, the window temperature is likely to show an increasingly superlinear rise as higher and higher orders of multiphonon intrinsic absorption are "switched on" by their stronger temperature dependence. ${ }^{1-4}$ Thus one will get "thermal runaway": Most probably, in a real system, this will occur at many localized sites, with the resultant local failures and stresses then interacting to produce overall failure.

In a recent paper $^{5}$ we presented calculations of the lowest-order intrinsic absorption for four alkali halides
( $\mathrm{NaCl}, \mathrm{KCl}, \mathrm{KBr}$, and $\mathrm{KI}$ ), chosen as model systems, to examine, among other things, any systematic trends in this absorption. At the longer wavelengths, the lowestorder absorption is determined by two-phonon difference processes $^{5}$ in which the third-order anharmonic terms in the lattice potential energy lead to the decay of the "virtual" transverse-optic phonon created by the radiation, the creation of one lattice phonon, and the destruction of another. The only general requirement on these processes, which also applies to the corresponding summation processes, is that the pairs of phonons involved have equal and opposite wave vectors. This ensures overall crystalmomentum conservation, since the optic phonon, created by the external radiation, has effectively zero crystal momentum.

Our results for these four alkali halides suggested a number of interesting trends whose general validity needs examination for a wide variety of systems. Unfortunately, this same work also demonstrated that the only reliable way to calculate the long-wavelength difference absorption is by direct summation of all contributions, wave vector by wave vector, over the Brillouin zone. Although symmetry allows one to reduce this summation to some degree, one is still required to use a very dense sample of wave vectors to obtain reliable values for the very-longwavelength absorption. One is thus faced with a major computational problem. This is compounded by the number of parameters available for variation in all but the simplest lattice-dynamical models. We resolved these problems by deliberately taking the simplest possible models and studying their absorption properties as their parameters are systematically varied. This we could do using an LSI 11/02 microcomputer, thus obviating the computational problem, since such a machine could be dedicated for long periods of time to this problem at only nominal cost. There was also the added advantage of 
direct interfacing to a highly flexible graphics system.

The next stage, to be described in detail in the body of this paper, was a comparison of the present "model" results to those for the four alkali halides studied earlier ${ }^{5}$ leading to the broad conclusion that the present model systems show the same range of behavior as the real systems. Finally, we proceeded to use the present approach to model the simple ceramics $\mathrm{MgO}$ and SrO.

\section{NATURE OF THE MODELS}

Initially we decided to study a two-dimensional system. Evidently this is something of a pedagogic exercise, but a comparison between the results obtained and those derived for the analogous three-dimensional systems is sufficiently interesting to warrant the presentation of some examples. The actual model used was a square lattice, composed of two interpenetrating face-centered square lattices ( 1 and 2 ), with atomic masses $m_{1}, m_{2}$, and with first- and second-neighbor linear springs resisting atomic displacements within the plane: Displacements normal to the plane were not permitted. The net effect is to produce a (001) plane of the sodium chloride structure. The subsequent three-dimensional studies were carried out on the sodium chloride structure itself, obtained by stacking these (001) planes on top of one another and adding in first- and second-neighbor spring constants between adjacent layers. For clarity, diagrams of these structures are shown in Fig. 1 (type-1 atoms, solid circles; type- 2 atoms, open circles).

These harmonic models were used to generate the normal-mode eigenfrequencies $\omega(\vec{q}, j)$ and eigenvectors $\overrightarrow{\mathrm{e}}(k \mid \overrightarrow{\mathrm{q}}, j)$ (“eigendata") used to compute the frequencydependent damping caused by third-order lattice anharmonicity. This damping is directly related to the real and imaginary parts of the frequency-dependent dielectric constant $\epsilon^{\prime}(\Omega)$ and $\epsilon^{\prime \prime}(\Omega)$, respectively.

For an arbitrary frequency these relationships are somewhat complicated; but at frequencies much lower than the fundamental TO frequency $[\omega(\overrightarrow{0}, j)]$ drastic simplification is possible, since it is sufficient to approximate the real part of the dielectric constant by its static value $\epsilon^{\prime}(0)$. We then have the following relations for the extinction coefficient $\kappa(\Omega)$ [see Ref. 5, Eqs. (2)-(4)]:

$$
\kappa(\Omega)=\frac{\epsilon^{\prime \prime}(\Omega)}{2 \sqrt{\epsilon^{\prime}(0)}}=\frac{\epsilon^{\prime}(0)-\epsilon^{\prime}(\infty)}{\omega(\overrightarrow{0}, j) \sqrt{\epsilon^{\prime}(0)}} \Gamma(\overrightarrow{0}, j ; \Omega) .
$$

(a)

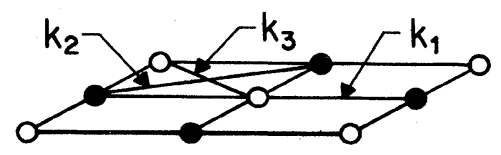

(b)

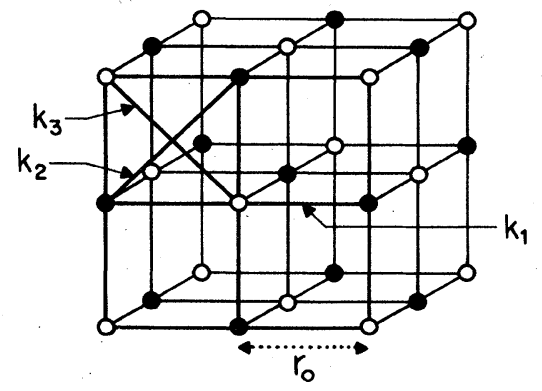

FIG. 1. Model lattices studied. (a) two-dimensional system; (b) three-dimensional system. In both cases the nearest- and next-nearest-neighbor springs are shown. (Type-1 atoms, solid circles; type- 2 atoms, open circles.)

The absorption coefficient $\alpha(\Omega)$ is given by

$$
\alpha(\Omega)=\frac{4 \pi \kappa(\Omega)}{\lambda} \quad\left[\lambda=\frac{2 \pi c}{\Omega}\right] .
$$

In view of the direct proportionality of $\kappa(\Omega)$ to the frequency-dependent damping $\Gamma(\overrightarrow{0}, j ; \Omega)$, the latter is a more convenient measure of absorption, since both will have the same frequency dependence. Furthermore, provided one considers only anharmonicity in first-neighbor bonds, one can reduce the expressions for $\Gamma(\overrightarrow{0}, j ; \Omega)$ and $\kappa(\Omega)$ to a product of a material-dependent constant and a Brillouin-zone sum which is a universal function depending only on force constants, mass ratio, and on reduced temperature. As a consequence, once this second function has been calculated, one can predict the behavior of very different systems simply by changing the prefactor.

If $r_{0}$ is the nearest-neighbor distance, $\psi^{\prime \prime \prime}\left(r_{0}\right)$ the third derivative of the first-neighbor potential, $n(\overrightarrow{\mathrm{q}}, j)$ the number of thermally excited phonons for the $(\vec{q}, j)$ mode and $N$ the number of unit cells; the explicit expression for $\Gamma(\overrightarrow{0}, j ; \Omega)^{5,6}$ is

$$
\begin{aligned}
& \Gamma(\overrightarrow{0}, j ; \Omega)=\frac{\pi \hbar\left(m_{1}+m_{2}\right)}{4 \omega(\overrightarrow{0}, j) m_{1} m_{2}} \lim _{\Delta \rightarrow \infty \rightarrow 0} \frac{1}{N \Delta \Omega} \int_{\Omega}^{\Omega+\Delta \Omega} d \Omega^{\prime} \sum_{\overrightarrow{\mathrm{q}}, j j^{\prime}}\left\{\psi^{\prime \prime \prime}\left(r_{0}\right)\left[e_{x}(1 \mid \overrightarrow{\mathrm{q}}, j) e_{x}\left(2 \mid-\overrightarrow{\mathrm{q}}, j^{\prime}\right)-e_{x}(2 \mid \overrightarrow{\mathrm{q}}, j) e_{x}\left(1 \mid-\overrightarrow{\mathrm{q}}, j^{\prime}\right)\right]\right. \\
& \left.\times \sin \left(q_{x} r_{0}\right)\right\}^{2}\left[m_{1} m_{2} \omega(\overrightarrow{\mathrm{q}}, j) \omega\left(-\overrightarrow{\mathrm{q}}, j^{\prime}\right)\right]^{-1} \\
& \times\left\{\left[n(\overrightarrow{\mathrm{q}}, j)+n\left(-\overrightarrow{\mathrm{q}}, j^{\prime}\right)+1\right] \delta\left(\Omega^{\prime}-\omega(\overrightarrow{\mathrm{q}}, j)-\omega\left(-\overrightarrow{\mathrm{q}}, j^{\prime}\right)\right)\right. \\
& \left.+\left|n(\overrightarrow{\mathrm{q}}, j)-n\left(-\overrightarrow{\mathrm{q}}, j^{\prime}\right)\right| \delta\left(\Omega^{\prime}-\left|\omega(\overrightarrow{\mathrm{q}}, j)-\omega\left(-\overrightarrow{\mathrm{q}}, j^{\prime}\right)\right|\right)\right\} .
\end{aligned}
$$


This expression is true for any model: Different models of the same crystal give different results insofar as they give different eigendata. The difference between two and three dimensions lies in the nature of the summations over the Brillouin zone.

The main shortcoming of the present simplified models is that longitudinal-optic (LO) and transverse-optic (TO) vibrations at the zone center are degenerate: The splitting due to long-range Coulomb interaction in real ionic materials is absent. However, this defect is much less serious than it seems at first sight. The reason, as will be demonstrated explicitly later, is that the primary contributions to $\Gamma(\overrightarrow{0}, j ; \Omega)$ are due to zone-boundary phonons. For these short-wavelength phonons, there is no such unique distinction between Coulomb and short-range contributions to the frequencies: It is thus possible to obtain a reasonable fit to their behavior from a model containing (adjustable) short-range forces alone. However, in order to obtain the direct coupling between the external radiation field and the long-wavelength TO vibrations it is necessary that the two sublattices carry equal and opposite "effective" ionic charges. This can be achieved by using real material values for the dielectric constants in Eq. (1).
This does not affect one's ability to adjust the short-range force constants in the dynamical matrix to produce the best overall fit to the zone-boundary phonon frequencies.

\section{SPECIFIC CALCULATIONS FOR TWO-DIMENSIONAL LATTICES}

Since these systems are strictly model systems, the only functions worth calculating are the phonon dispersion curves (plots of angular frequency $\omega$ versus wave vector $\overrightarrow{\mathrm{q}}$ ); the frequency distribution function (number of normal modes per unit angular-frequency range), and the damping function $\Gamma(\overrightarrow{0}, j ; \Omega)$ defined by Eq. (3). These contain all the information relevant to our present discussion.

For the model systems shown in Fig. 1, the squares of the normal-mode frequencies for a given wave vector $\vec{q}$ are determined by the eigenvalue equation for the dynamical matrix $D_{\alpha \beta}^{i j}(\overrightarrow{\mathrm{q}})$; where $i$ and $j$ index the two sublattices, and $\alpha(\beta)$ are $x$ or $y$, (or $x, y$, or $z$ ) for the two (or three) Cartesian axes. The elements of $D_{\alpha \beta}^{i j}(\overrightarrow{\mathrm{q}})$ are as follows.

$$
\begin{aligned}
& \text { 3D: } \\
& \qquad D_{\alpha \alpha}^{11}(\overrightarrow{\mathrm{q}})=\frac{2}{m_{1}}\left(k_{1}+k_{2}\left\{2-\cos \left(q_{\alpha} r_{0}\right)\left[\cos \left(q_{\beta} r_{0}\right)+\cos \left(q_{\gamma} r_{0}\right)\right]\right\}\right), \alpha \neq \beta \neq \gamma \neq \alpha
\end{aligned}
$$

2D:

$$
D_{\alpha \alpha}^{11}(\overrightarrow{\mathrm{q}})=\frac{2}{m_{1}}\left\{k_{1}+k_{2}\left[1-\cos \left(q_{\alpha} r_{0}\right) \cos \left(q_{\beta} r_{0}\right)\right]\right\}, \alpha \neq \beta
$$

3D and 2D:

$$
D_{\alpha \beta}^{11}(\overrightarrow{\mathrm{q}})=\frac{2}{m_{1}}\left[k_{2} \sin \left(q_{\alpha} r_{0}\right) \sin \left(q_{\beta} r_{0}\right)\right], \quad \alpha \neq \beta
$$

3D:

$$
D_{\alpha \alpha}^{22}(\overrightarrow{\mathrm{q}})=\frac{2}{m_{2}}\left(k_{1}+k_{3}\left\{2-\cos \left(q_{\alpha} r_{0}\right)\left[\cos \left(q_{\beta} r_{0}\right)+\cos \left(q_{\gamma} r_{0}\right)\right]\right\}\right), \alpha \neq \beta \neq \gamma \neq \alpha
$$

2D:

$$
D_{\alpha \alpha}^{22}(\overrightarrow{\mathrm{q}})=\frac{2}{m_{2}}\left\{k_{1}+k_{3}\left[1-\cos \left(q_{\alpha} r_{0}\right) \cos \left(q_{\beta} r_{0}\right)\right]\right\}, \alpha \neq \beta
$$

3D and 2D:

$$
D_{\alpha \beta}^{22}(\overrightarrow{\mathrm{q}})=\frac{2}{m_{2}}\left[k_{3} \sin \left(q_{\alpha} r_{0}\right) \sin \left(q_{\beta} r_{0}\right)\right], \alpha \neq \beta
$$

3D and 2D:

$$
D_{\alpha \alpha}^{12}(\overrightarrow{\mathrm{q}})=\frac{-2}{\left(m_{1} m_{2}\right)^{1 / 2}}\left[k_{1} \cos \left(q_{\alpha} r_{0}\right)\right], \quad D_{\alpha \beta}^{12}(\overrightarrow{\mathrm{q}})=0, \alpha \neq \beta .
$$

In Eqs. (4)-(10), $k_{1}, k_{2}$, and $k_{3}$ are, respectively, the first-neighbor, and the two distinct second-neighbor (11 and 22) spring constants.

The first Brillouin zone for the two-dimensional lattice is a square and we chose, as a representative display, plots of the dispersion curves as $\vec{q}$ runs along the diagonal from the zone center $\Gamma$ to the zone corner $M$; then along the zone edge from $M$ to $X$, the edge center; and, finally, back from $X$ to $\Gamma$ perpendicular to the edge.

The area of the zone bounded by these lines constitutes the irreducible $\frac{1}{8}$ of the zone: Eigendata for the remainder of the zone can be generated from those in the irreducible area by simple symmetry operations and do not have to be calculated separately. However, to calcu- 
late the frequency distribution and $\Gamma(\overrightarrow{0}, j ; \Omega)$ it is necessary to derive the eigendata for wave vectors throughout the irreducible area. In practice, this means computing them for a sufficiently fine regular mesh of points and binning the associated contributions, either to the distribution or to $\Gamma(\overrightarrow{0}, j ; \Omega)$ into small but finite frequency bins. Their width is chosen to be some fixed fraction $\left(\sim 10^{-2}\right)$ of the maximum frequency for each function. The ability to handle the problem on a microcomputer enabled us to step up the density of the wave-vector sample and narrow the bin widths to the point where we could obtain nearcontinuous curves with histogram "chatter" almost eliminated.

From the form of the dynamical matrix it is evident that one can factor out $k_{1} / m_{1}$, and thus work in "natural" units of $K_{1}=\left(k_{1} / k_{1}\right)=1, K_{2}=\left(k_{2} / k_{1}\right), K_{3}$ $=\left(k_{3} / k_{1}\right), \quad \boldsymbol{M}_{2}=\left(m_{2} / m_{1}\right), \quad \boldsymbol{M}_{1}=\left(m_{1} / m_{1}\right)=1, \quad$ and $\Omega(\overrightarrow{\mathrm{q}}, j)=\omega(\overrightarrow{\mathrm{q}}, j) /\left(k_{1} / m_{1}\right)^{1 / 2}$. This is the convention we adopted, and most of our results (both for two- and three-dimensional systems) will be presented in this form:
The only exceptions are the calculations of $\epsilon^{\prime \prime}(\Omega)$ and $\Gamma(\overrightarrow{0}, j ; \Omega)$ for specific three-dimensional systems. Otherwise our $\Gamma(\overrightarrow{0}, j ; \Omega)$ values are in natural units, without the prefactor in Eq. (3) included and $\psi^{\prime \prime \prime}\left(r_{0}\right)$ set equal to unity. This also means that the quantity $\hbar / k T(k$, Boltzmann's constant; $T$ the absolute temperature), which occurs in the phonon population numbers and has dimensions $(\mathrm{rad} / \mathrm{sec})^{-1}$, is also specified in natural units.

In Fig. 2 we show sample results for a pair of twodimensional lattices, one with equal masses and the other with 1:2 mass ratio. Phonon-dispersion curves, density, and damping are shown for each case. In the case of the damping the "temperature" has been determined by setting $\hbar / k T=1$ : This is approximately the equivalent of room temperature if material parameters appropriate to a typical ceramic are used, and $\sim 50-100 \mathrm{~K}$ for typical alkali halide parameters. The ratio of first- to secondneighbor spring constants was taken as $4: 1$, which the three-dimensional studies (to be described subsequently) have shown to be reasonable to approximate real systems.
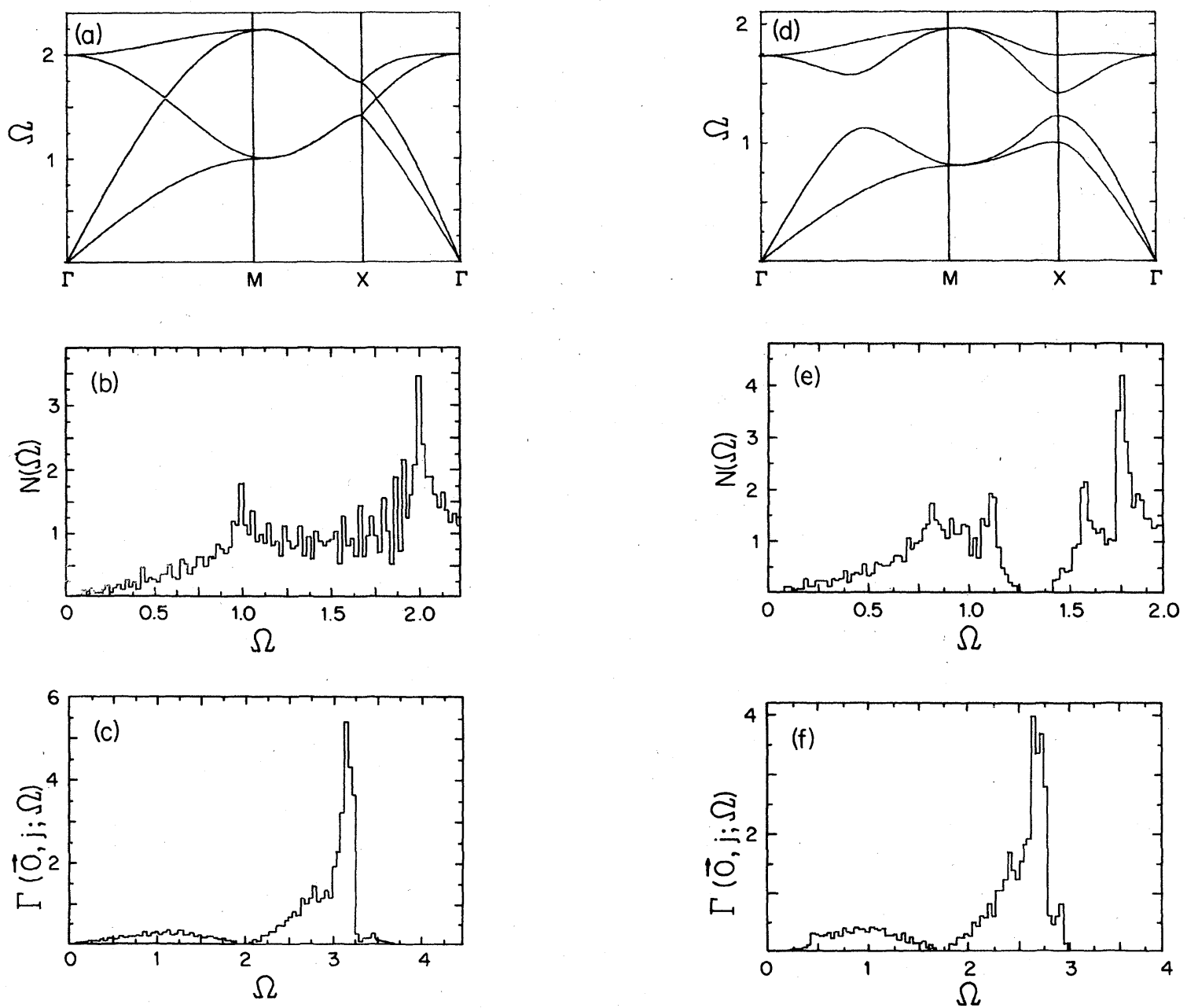

FIG. 2. Data for the two-dimensional lattices. (a) dispersion curves ( $\Omega$ versus $\overrightarrow{\mathrm{q}}$ ) for $M_{1}=M_{2}=1, K_{1}=1, K_{2}=K_{3}=\frac{1}{4}$; (b) corresponding frequency distribution; (c) corresponding damping function; (d) dispersion curves for $M_{1}=1, M_{2}=2, K_{1}=1, K_{2}=K_{3}=\frac{1}{4}$; (e) corresponding frequency distribution; and (f) corresponding damping function. In all cases the frequencies $\Omega$ are in "natural" units (see text). The vertical scales on the frequency distributions and dampings are in arbitrary units, however, these are equal for the same functions. The two dampings are computed for $\hbar / k T=1$ in natural units (see text). 
Most notable, is the appearance of a gap in the frequency spectrum of the lattice with unequal masses, whose presence is also manifest in the clear separation between the optic and acoustic branches of the associated dispersion curves. Less dramatic, but of much more practical significance, is the strong suppression of the damping function at low frequencies when the masses are unequal. A similar effect was found during our earlier studies on real alkali halides, ${ }^{5}$ and it is gratifying to find that the present simplified models reproduce this behavior, even when they are reduced to two-dimensional systems. Also, it should be noted that the width of this "window" is significantly greater than the gap in the frequency distribution. Indeed, it appears to correspond to the spacing between the highest-frequency peak in the acoustic mode spectrum and the lowest-frequency peak in the optic mode spectrum. The probable reason for this is that the window appears when energy conservation for difference processes (which are the only processes allowed at low frequencies), "freezes out" interband contributions involving optic phonon creation and acoustic phonon destruction. Below this cutoff the only processes allowed are intraband processes: For these, when the ionic masses are markedly different, the eigenvector products in Eq. (3) are necessarily small, since the motions of the optic (acoustic) branches are primarily confined to the lighter (heavier) sublattices.

Also worthy of note is the relatively featureless structure of the whole difference-band region of the damping for both lattices. Apart from the low-frequency window when the masses are unequal, both damping functions appear as smooth "bell-like" shapes; again this is remarkably similar to the results for real alkali halides. ${ }^{5-8}$

The only truly unrealistic behavior of these damping functions is that which is manifest in the summation region as sudden finite jumps. These are genuine (i.e., they are not an artifact of the use of a finite sample of wave vectors) and simply reflect the effect of two-dimensional van Hove ${ }^{9}$ singularities in the two-phonon density of states. These occur whenever

$$
\vec{\nabla}_{\overrightarrow{\mathrm{q}}}[\omega(\overrightarrow{\mathrm{q}}, j)+\omega(-\overrightarrow{\mathrm{q}}, j)]=\overrightarrow{0}
$$

and produce associated finite discontinuities (or logarithmic singularities) in this density. These are analogous to the corresponding singularities in the single-phonon frequency spectra, which are also apparent in Fig. 2. The only difference is that they can be more numerous, since only the sum or difference in Eq. (11) is required to vanish: The individual terms can be finite. It is thus possible to have two-phonon singularities at points in reciprocal space at which there are no associated one-phonon singularities.

The similarity between these results for a twodimensional system and those for real three-dimensional systems is highly encouraging. Particularly gratifying is the agreement in the low-frequency region, since it implies that one can use the present simplified model, or more properly its three-dimensional counterpart, to obtain a definitive map of the damping at very low frequencies. This is possible because one can enhance the density of the wave-vector sample to the point where histogram "chatter" is smoothed out in this low-frequency region. For the "typical" density and bin width, used in generating Fig. 2, the histogram steps are too coarse to define the very-low-frequency region adequately. Specifically, one can see that the bin width used in Fig. 2 is $\sim 1 \%$ of the fundamental dispersion frequency, while millimeter electromagnetic frequencies are also $\sim 1 \%$ of this frequency. Thus one needs a much smaller bin width if one is to define the damping at such low frequencies. However, any attempt to "re-bin" the data of Fig. 2 in such a fashion will simply produce violent "chatter" in the resulting histograms, particularly in the very-low-frequency region where the contributions are inherently small. To remedy this it is necessary to increase the density of wave vectors employed, and calculations were made for both equal and unequal masses when the sample density was increased by $(8)^{2}$. This increases the computer time required by approximately the same factor. With a dedicated microcomputer this presents no problem-if necessary it can be left to run overnight. Since these results are qualitatively very similar to those for the realistic three-dimensional models, to be presented later, a full graphical display would be needlessly repetitious, and it suffices to describe the salient features of the results. However, a fuller report of this work, containing a more comprehensive display of these and other results we shall describe subsequently, is available on request. The enhanced sample of wave vectors is sufficient to provide "chatter-free" histograms of the damping function between 0 and 0.1 natural frequency units when this range is divided into 50 bins. From these results, the dramatic suppression of the damping for the case of unequal masses, indicated by Fig. 2, is clearly apparent. Moreover, the marked nonlinearity of its rise with frequency for this case is also obvious. This is entirely consistent with the damping in this region arising from near-forbidden intraband difference processes involving pairs of near-degenerate phonons close to symmetry points (lines) in reciprocal space. This "near-forbiddenness" can have two origins: The first, alluded to earlier, is due to the smallness of the eigenvector products in Eq. (3); the second may arise from the processes involved being strictly forbidden at (on) a symmetry point (line) (e.g., a longitudinal-transverse combination involving two phonons propagating along a symmetry axis).

\section{SURVEY CALCULATIONS FOR THREE-DIMENSIONAL SYSTEMS}

In the light of these encouraging results for twodimensional systems, we proceeded with a series of systematic studies for three-dimensional models. The models studied were three-dimensional equivalents of those studied in two dimensions. However, in order to determine the trend of the results, we examined those obtained for four relative strengths of first- to second-neighbor force constants $(1: 1,2: 1,3: 1$, and 4:1), while keeping the latter equal for both types of second-neighbor. In each case, results were obtained for both equal masses and a 1:2 mass ratio; again the "temperature" was defined by requiring $\hbar / k T$ to be unity.

On the basis of these results, we selected the lattices 
having a 4:1 ratio for first- to second-neighbor force constants as being the most "typical" of a real system, and the resultant dispersion curves, frequency distributions, and damping functions are shown in Fig. 3. This was a somewhat arbitrary choice; however, particularly in the low-frequency region, we found that the damping (our major concern) is relatively insensitive to this ratio; as long as it is substantially less than unity, as must be the case for any physically reasonable interatomic potential. Given these facts, we chose not to vary $K_{2}$ and $K_{3}$ independently and proceeded to a detailed study of the lowfrequency parts of the damping for these systems, using a very fine mesh of sample wave vectors. These last calculations are substantially more time consuming than the analogous two-dimensional calculations, as the time required rises as the cube, rather than the square, of the sample density.

For all force-constant ratios, a low-frequency window appears in the damping when the mass ratio is $1: 2$. This, and the nonlinear rise with frequency, are most clearly ap- parent in the expanded displays of this region for our selected models, which mirror those for two-dimensional systems. Again a fuller presentation of these data, along with results for other force constant ratios, is available on request.

The overall appearance of the damping functions, particularly in the difference regions, is surprisingly similar to the two-dimensional results. The main difference is that the discontinuities present for the two-dimensional examples, principally in the summation region, are removed and replaced by well-behaved maxima and minima. Moreover, there is a strong general similarity to the results for real alkali halides, ${ }^{5-7}$ particularly marked (to the extent of near-identity) in the low-frequency difference region, but present even in the more highly structured summation region.

In the case of the very-low-frequency damping it is also of particular interest to determine from which ranges of the vibrational frequency distribution the contributions to the damping arise. We therefore wrote a subprogram that
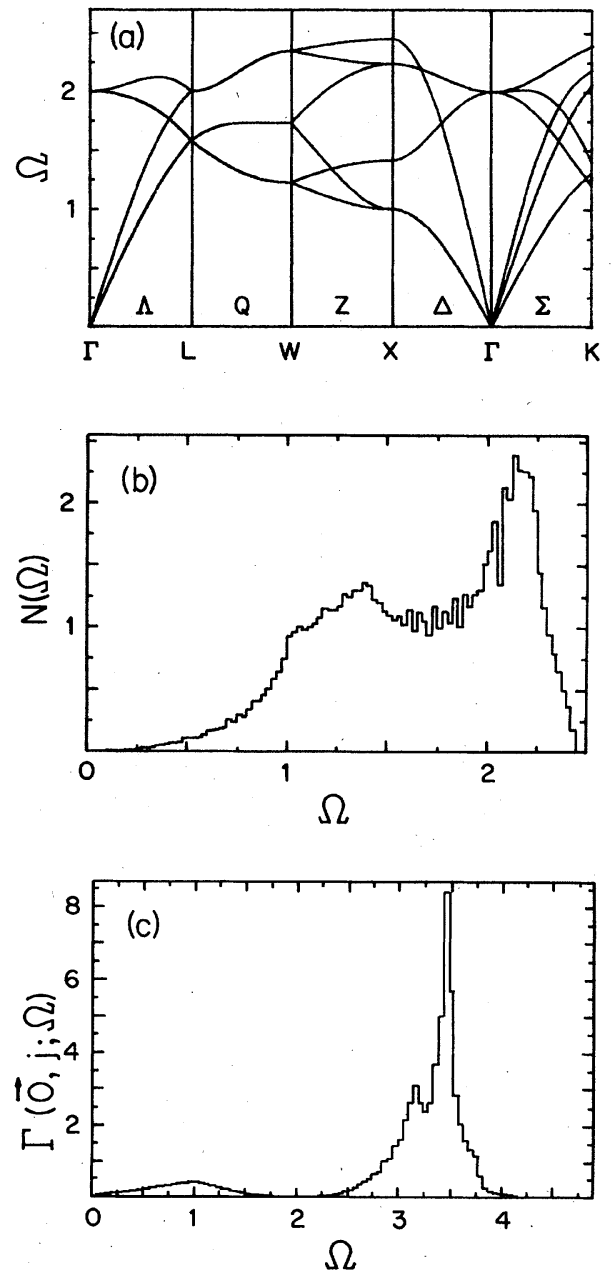
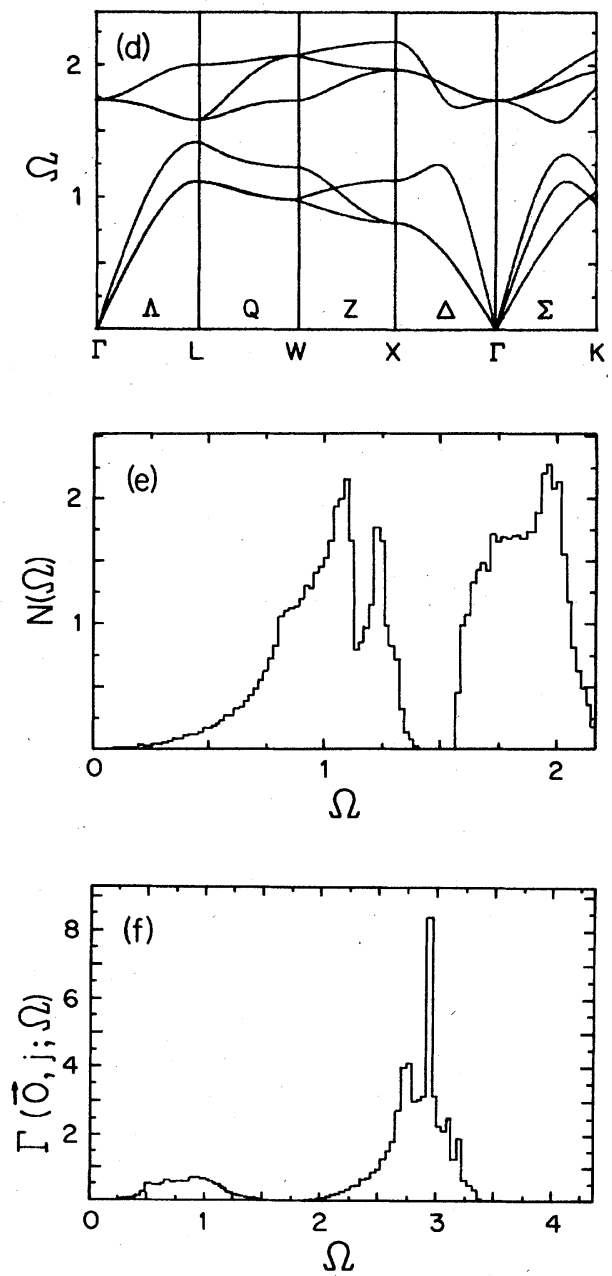

FIG. 3. Data for the three-dimensional lattices. (a) dispersion curves ( $\Omega$ versus $\overrightarrow{\mathrm{q}}$ ) for $M_{1}=M_{2}=1, K_{1}=1, K_{2}=K_{3}=\frac{1}{4}$; (b) corresponding frequency distribution; (c) corresponding damping function; (d) dispersion curves for $M_{1}=1, M_{2}=2, K_{1}=1, K_{2}=K_{3}=\frac{1}{4}$; (e) corresponding frequency distributions; and (f) corresponding damping function. In all cases the frequencies $\Omega$ are in "natural" units (see text). The vertical scales on the frequency distributions and dampings are in arbitrary units, however, these are equal for the same functions. The dampings are computed for $\hbar / k T=1$ in natural units (see text). 
counted one unit into each bin of the frequencydistribution histogram for each mode within that bin range that contributed to the low-frequency damping. Results were obtained using this program for our selected two- and three-dimensional models and superposed on the actual frequency distribution. It was found that the contributions are sharply peaked in specific regions of the frequency spectrum. This is particularly marked in the two-dimensional cases, and reflects the sharp "pile up" of contributions from areas of near-degeneracy close to singularities associated with symmetry lines (points), mainly on the zone boundary. As was observed earlier, these are much stronger in two rather than three dimensions. For the three-dimensional systems, the contributions are more spread out, but this probably mainly reflects the greater number of singularities when the dispersion surfaces involve six rather than four branches and have three-dimensional topology. Since these results are qualitatively similar to those to be presented subsequently for realistic systems we do not display them at this point, but they are available on request.

As an ancillary calculation we also examined the overall temperature dependence of the damping functions. In Fig. 4 we show superposed the results for a range of
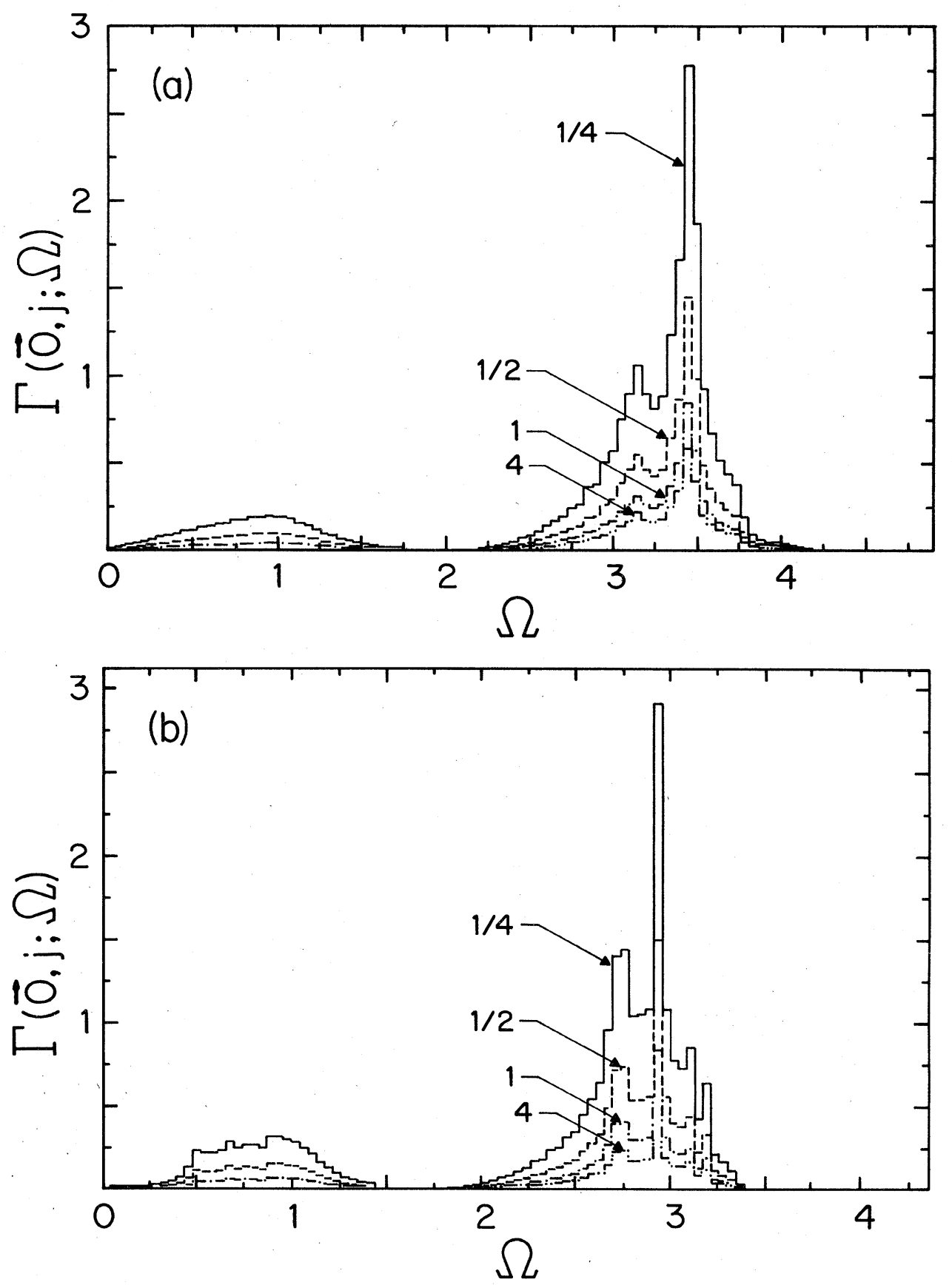

FIG. 4. Superpositions of the dampings at different temperatures for our two selected three-dimensional lattices ( $K_{1}=1$, $K_{2}=K_{3}=\frac{1}{4}$ ). (a) $M_{1}=M_{2}=1$; (b) $M_{1}=1, M_{2}=2$. Values of $\hbar / k T$ in natural units (see text) are indicated on each histogram. 
values of $\hbar / k T$ from 4 to $\frac{1}{4}$ for our two standard threedimensional systems. The sharp drop at lower temperatures in the difference band (low frequency) region is readily apparent, as is the asymptotic linear behavior at all frequencies at the higher temperatures.

\section{ABSOLUTE VALUES OF THE LOSSES FOR MgO AND SrO}

In order to provide some quantitative insight into the absolute values of absorptions (losses) to be expected in typical ceramic materials we have taken two specific cases, chosen to represent magnesium oxide $(\mathrm{MgO})$ and strontium oxide (SrO). Within the present model we can only mimic these by varying the mass ratios and relative magnitudes of the first- and second-neighbor force constants. However, we believe this to be adequate to illustrate the overall trend, and to establish that the heavier softer material does indeed show a lower absorption level in the millimeter and submillimeter frequency range. In this last context we felt it best to exclude the heaviest alkaline earth oxide, barium oxide, as it appears to have an anomalously high static dielectric constant $\left[\epsilon^{\prime}(0) \sim 30\right]$ as compared with ceramics of practical interest.

In order to calculate the appropriate potential derivatives in Eq. (3), we followed a rather different procedure from that used in Ref. 5. Specifically, we took the parameters for the short-range potential between first neighbors from the compilation by Stoneham ${ }^{10}$ and added to this their Coulomb interaction, assuming full ionicity (i.e., ionic charges of plus or minus twice the electronic charge). Thus we considered only the third derivative of the firstneighbor potential to be significant and included both Coulomb and short-range components. This approximation [implicit in Eq. (3)] is better than it seems at first sight, since it can be shown that second-neighbor contributions to the damping are identically zero. In this situation it would appear that anything other than firstneighbor contributions is genuinely insignificant.

Calculations of the low-frequency damping were then made for two three-dimensional lattices: one having a mass ratio of 1:1.5 (corresponding to $\mathrm{MgO}$ and one having a mass ratio of 1:5 (corresponding to $\mathrm{SrO}$ ). In order that the results be strictly comparable, the value of $\hbar / k T$ was set at 0.7 for the second system while the "standard value" of unity was used for the first system. Both systems are then at approximately $310 \mathrm{~K}$. In each case the ratio of first- to second-neighbor force constants was given the standard value of $4: 1$. The resulting lowfrequency dampings and contributions (computed as described earlier for the standard runs) are shown in Fig. 5. However, for both the damping histograms, the scales are labeled in absolute units. The conversion was made using the third derivatives computed from Stoneham's tabulation $^{10}$ and their values are indicated in the caption for Fig. 5. Also, on the right-hand vertical axis are shown the associated values of the imaginary part of the dielectric constant $\epsilon^{\prime \prime}(\Omega)$, computed neglecting any variation with frequency of the real part, an approximation which is certainly accurate at these low frequencies. It is at once apparent that the lighter material shows the dominant
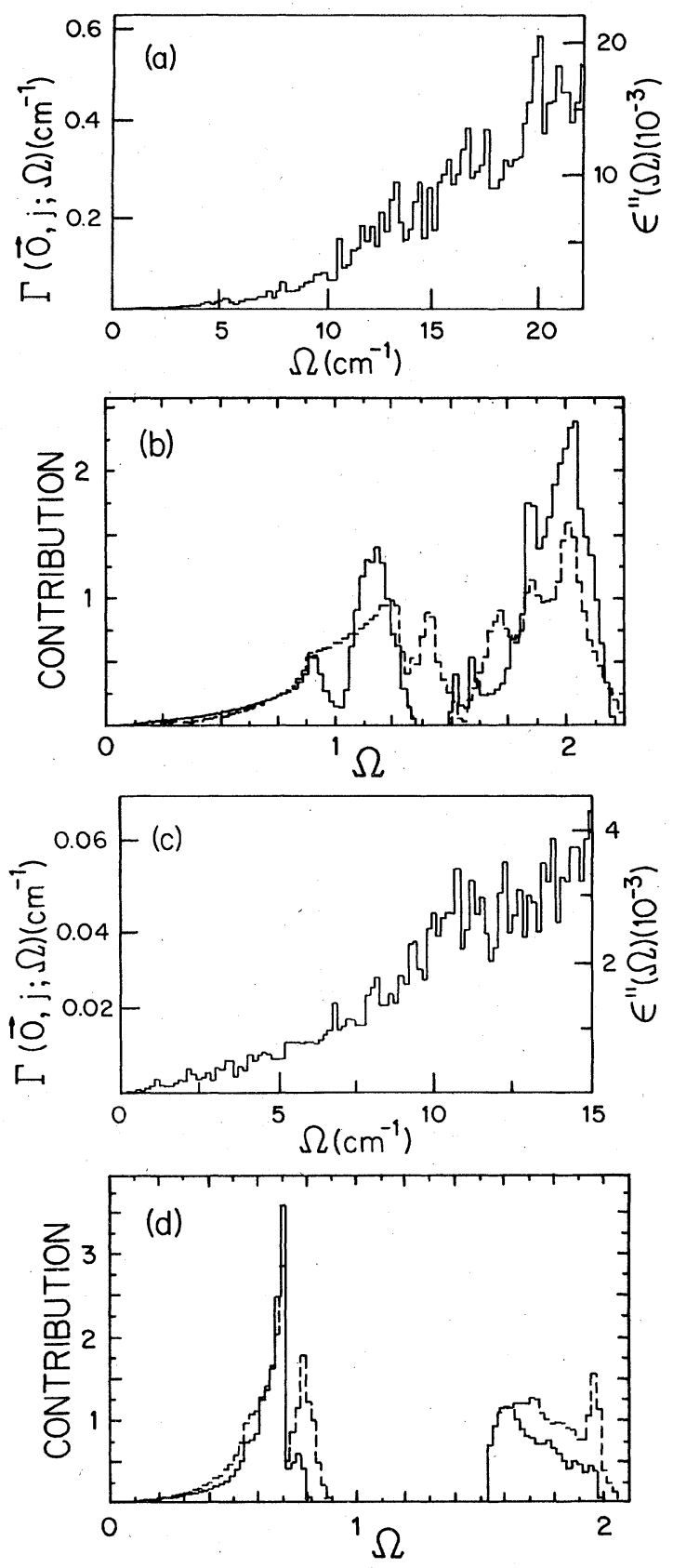

FIG. 5. Absolute values for low-frequency dampings and imaginary dielectric functions, $\epsilon$ " $(\Omega)$, for two "real" systems, together with the corresponding "contributions." (a) $\mathrm{MgO}$ damping and loss: $M_{1}=1, M_{2}=1.5, K_{1}=1, K_{2}=K_{3}=\frac{1}{4}$, $T=310 \mathrm{~K}\left(\hbar / k T=1\right.$ in natural units), $\psi^{\prime \prime \prime}\left(r_{0}\right)=50.488 \times 10^{12}$ dynes $/ \mathrm{cm}^{2}$. (b) $\mathrm{MgO}$ contribution (full line): superposed, and scaled to be comparable, the $\mathrm{MgO}$ frequency distribution (broken line). Vertical scale (corresponding to the contribution) is arbitrary, however, $\Omega$ is given in multiples of $4.11 \times 10^{13}$ radians/sec (the unit of natural frequency). (c) SrO damping and loss: $M_{1}=1, M_{2}=5, K_{1}=1, K_{2}=K_{3}=\frac{1}{4}, T=310 \mathrm{~K}$ $\left(\hbar / k T=0.7\right.$ in natural units), $\psi^{\prime \prime \prime}\left(r_{0}\right)=24.440 \times 10^{12}$ dynes $/ \mathrm{cm}^{2}$. (d) SrO contribution (full line): superposed, and scaled to be comparable, the SrO frequency distribution. The vertical scale (corresponding to the contribution) is arbitrary; however, $\Omega$ is given in multiples of $2.85 \times 10^{13}$ radians $/ \mathrm{sec}$ (the unit of natural frequency). 
loss, with values almost an order of magnitude larger over the whole frequency range. Since we are now discussing absolute magnitudes, it is important to realize that additional factors have come into play. The lower loss in the heavier material results not only from the strong suppression of the low-frequency damping, due to the eigenvector properties discussed earlier, but also because the combination of heavier masses and a softer potential overwhelms the strong enhancement due to the lower phonon frequencies in the heavier system.

As far as comparison with experiment is concerned, there appears to be very little optical data for such systems at millimeter and submillimeter frequencies. The only recent data appear to be those of Afsar and Button, ${ }^{11}$ who present results for various glass ceramics, alumina, and beryllium oxide. All seem to show similar losses, which are of the order of two to three times the values we have deduced for $\mathrm{MgO}$, to which, in terms of atomic mass ratio, they are most comparable. However, it is clear from their data that there is present in their specimens some extrinsic loss which is certainly dominant below $\sim 5$ $\mathrm{cm}^{-1}$ and is probably present throughout the spectral region $\left(5-14 \mathrm{~cm}^{-1}\right)$ covered by their data. If one postulates that the rising absorption they find at higher frequencies is due to intrinsic loss superposed on a reasonably flat background of extrinsic loss, then these excess losses are, to within $50 \%$, in agreement with our results for $\mathrm{MgO}$, which would argue both for their intrinsic nature and the usefulness of our theory. At present, this is a very speculative conclusion. An obvious and definitive test would be to repeat Afșar and Button's measurements ${ }^{11}$ for a range of specimen temperatures. Such a test would also verify whether or not the two-phonon loss we have studied is the dominant intrinsic loss mechanism or whether three-phonon losses have to be included. In the case of materials in which the atomic masses are comparable, earlier work ${ }^{8,12}$ on alkali halides has shown this to be unlikely. For ceramics it seems even less likely, since their markedly higher phonon frequencies tend to reduce the number of thermally excited quanta, and thus would further suppress three-phonon processes as compared with two-phonon processes.

\section{CONCLUSIONS}

We have demonstrated, by means of a number of simple model calculations, that the intrinsic losses in ceramic dielectrics at millimeter and submillimeter frequencies are subject to the same general rules as losses in softer ionic materials, such as alkali halides. Specifically, we have confirmed the existence of a window effect, whereby heavier materials with a large disparity between the masses of the constituent ions show order of magnitude lower intrinsic losses in the millimeter spectral region than lighter materials in which the ionic masses are more nearly equal. This occurs despite the fact that the heavier materials tend to be significantly softer. Given the simplified nature of our calculations, the absolute values we predict for these losses appear to be in reasonable accord with estimates of the intrinsic losses in real systems.

One final point of general interest, which emerges from this and earlier studies, is a probable explanation for the general "anomalous" behavior of observed dampings in the vicinity of the fundamental resonance. At high temperatures, these quantities appear to vary quadratically rather than linearly with temperature, implying threerather than two-phonon decay. The reason, which appears to be generally valid, is that maximum of the twophonon difference band occurs at the zone-center LO mode frequency, while the lowest-frequency summation process occurs at the zone-center TO mode frequency. Thus only the tails of the difference processes are available for two-phonon decay and, again typically, these tails drop steeply as the TO phonon frequency is approached. Thus two-phonon decay is strongly inhibited in the vicinity of the fundamental resonance (i.e., the TO phonon frequency), and three-phonon decay becomes dominant. This behavior, and its general validity, are due to its origin in the much more stringent selection rules for twophonon decay. Specifically, energy conservation and the requirement that the two phonons have equal and opposite wave vectors virtually ensures that the maximum of the difference band and the minimum of the summation band involve processes in which optical phonons of approximately zero wave vector are created and approximately zero wave-vector acoustic phonons are, respectively, destroyed and created. For the difference band maximum it is an LO phonon that is created, while for the summation band minimum it is a TO phonon that is created. Since these processes are near-forbidden, twophonon decay is strongly suppressed. While it is possible to conceive of phonon-dispersion curves, that violate these conditions (e.g., if a soft TO mode is present) they do not represent the behavior typical of these curves for simple binary compounds. ${ }^{13,14}$ For three-phonon decay there are no such inhibitions, since processes in which two zoneboundary phonons of near-equal frequency are, respectively, created and destroyed, and one zone-center TO phonon is created, will provide a high density of final states and an associated large damping in the vicinity of the fundamental resonance.

\section{ACKNOWLEDGMENTS}

This work was supported by the U.S. Office of Naval Research under Contract No. N00014-80-C-0518. We are also indebted to the solid-state experimental group at the University of Nebraska, in particular, Professor R. D. Kirby, for access to their microcomputer facilities.
${ }^{1}$ L. Genzel, H. Happ, and R. Weber, Z. Phys. (Leipzig) 154, 13 (1959).

${ }^{2}$ H. Dötsch and H. Happ, Z. Phys. (Leipzig) 177, 360 (1964).

${ }^{3}$ R. Stolen and K. Dransfeld, Phys. Rev. 139A, 1295 (1965).
4J. C. Owens, Phys. Rev. 181, 122 (1969).

5J. R. Hardy and A. M. Karo, Phys. Rev. B 26, 3327 (1982).

6J. E. Eldridge and R. Howard, Phys. Rev. B 7, 4652 (1973).

7J. E. Eldridge and K. A. Kembry, Phys. Rev. B 8, 746 (1973). 
8J. E. Eldridge and P. R. Staal, Phys. Rev. B 16, 4608 (1977).

${ }^{9}$ L. van Hove, Phys. Rev. 89, 1189 (1953).

${ }^{10}$ A. M. Stoneham, Atomic Energy Research Establishment Report No. R9598, 1979 (unpublished), p. 92.

${ }_{11}$ M. N. Afsar and K. J. Button (unpublished).

12J. E. Eldridge and P. R. Staal, Phys. Rev. B 16, 3834 (1977).
${ }^{13}$ H. Bilz and W. Kress, Phonon Dispersion Relations in Insulators (Springer, Berlin, 1979) p. 28.

14J. R. Hardy and A. M. Karo, The Lattice Dynamics and Statics of Alkali Halide Crystals (Plenum, New York, 1979), p. 170. 